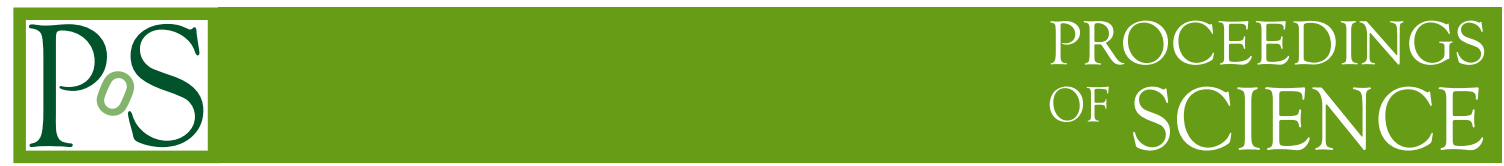

\title{
Precise luminosity determination at CMS
}

\author{
M.M.A. Gadallah ${ }^{a, b, *}$ on behalf of the CMS Collaboration \\ ${ }^{a}$ MTA-ELTE Lendület CMS Particle and Nuclear Physics Group, Eötvös Loránd University, Budapest, \\ Hungary \\ ${ }^{b}$ Physics Department, Assiut University, Assiut, Egypt \\ E-mail: mahmoud.gadallah@cern.ch
}

Precise luminosity calibration at bunched-beam hadron colliders like the Large Hadron Collider (LHC) is critical to determine fundamental parameters of the standard model and to discover or constrain beyond-the-standard-model phenomena. The results of luminosity determination at LHC interaction point 5 with the Compact Muon Solenoid (CMS) detector, using proton-proton (pp) collisions in 2018, is reported. The calibration is derived by analyzing the data of the van der Meer (vdM) scan program. The total uncertainty is $2.5 \%$ (similar to previous years) and is dominated by the contributions due to the $x-y$ factorization assumption and luminometer linearity.

The Eighth Annual Conference on Large Hadron Collider Physics-LHCP2020 25-30 May, 2020

Online

\footnotetext{
${ }^{*}$ Speaker
} 


\section{Introduction}

Several systems ("luminometers") are exploited to measure luminosity at the Compact Muon Solenoid (CMS) detector [1]. The Pixel Luminosity Telescope and the Fast Beam Conditions Monitor are dedicated systems for luminosity measurement. They, as well as the system dedicated to luminosity and installed as part of the Hadron Forward Calorimeter (HF), use a separate data acquisition (DAQ) system which operates independently of the main CMS readout. HF provides luminosity measurements with two algorithms - one based on the fraction of occupied towers (HFOC), and the other on the sum of the transverse energy(HFET). Luminosity measurements can be thus provided regardless of the operating state of the rest of CMS. In addition, three other methods, based on counting muon stubs in the Drift Tubes (DT), pixel cluster counting (PCC), and vertex counting using the inner tracker information, are employed to perform luminosity measurements relying on the main CMS DAQ system. Finally, the RAMSES detectors, which are part of the LHC environmental protection and radiation monitoring system, are also used. [2]

Each luminometer reads out a rate of specific observables (hits, tracks, clusters, etc.). This rate, $R(t)$, should be proportional to the instantaneous luminosity, $\mathcal{L}(t)$, with the constant of proportionality given by the visible cross section, $\sigma_{\text {vis }}$ :

$$
R(t)=\mathcal{L}(t) \sigma_{\text {vis }}
$$

The calibration constant $\sigma_{\text {vis }}$ is determined using van der Meer (vdM) beam separation scans using the formula

$$
\sigma_{\text {vis }}=\frac{2 \pi \Sigma_{x} \Sigma_{y}}{N_{1} N_{2} f} R_{\text {peak }}
$$

where it is assumed that the bunch proton density function is factorizable into independent $x$ and $y$ terms, $N_{1}$ and $N_{2}$ are the number of protons in the two colliding bunches, $f$ is the orbit frequency, $\Sigma_{\mathrm{x}}, \Sigma_{\mathrm{y}}$ are the beam overlap widths in the $x$ and $y$ directions and $R_{\text {peak }}$ is the rate measured at zero transverse beam separation. The measured value of $\sigma_{\mathrm{vis}}$ is then used during physics fills to calculate the per bunch $\mathcal{L}(t)$. Integrating $\mathcal{L}(t)$ over colliding bunch pairs and time gives the integrated luminosity used in physics studies. This report shows the results of luminosity determination at LHC interaction point 5 with the CMS detector, using 2018 proton-proton (pp) collisions at $\sqrt{s}=$ $13 \mathrm{TeV}$.

\section{Systematic uncertainties}

As described in Refs. [2-4], there are several systematic effects called "normalization uncertainties" which affect $\sigma_{\text {vis }}$ extracted from the vdM scan analysis. "Integration uncertainties", on the other hand, arise from detector operations over the course of the year, as well as from the application of the calibration from the special vdM fill conditions to the physics ones.

\subsection{Normalization uncertainties}

To correct possible differences between the actual and nominal beam separations during the scans, length scale calibration (LSC) is performed using two different methods both relying on the measurement of the luminous region position based on vertex reconstruction in the CMS tracker. 
The final correction and uncertainty in $\sigma_{\text {vis }}$ due to the length scale is then evaluated by combining the two independent scan results, illustrated on Fig. 1.
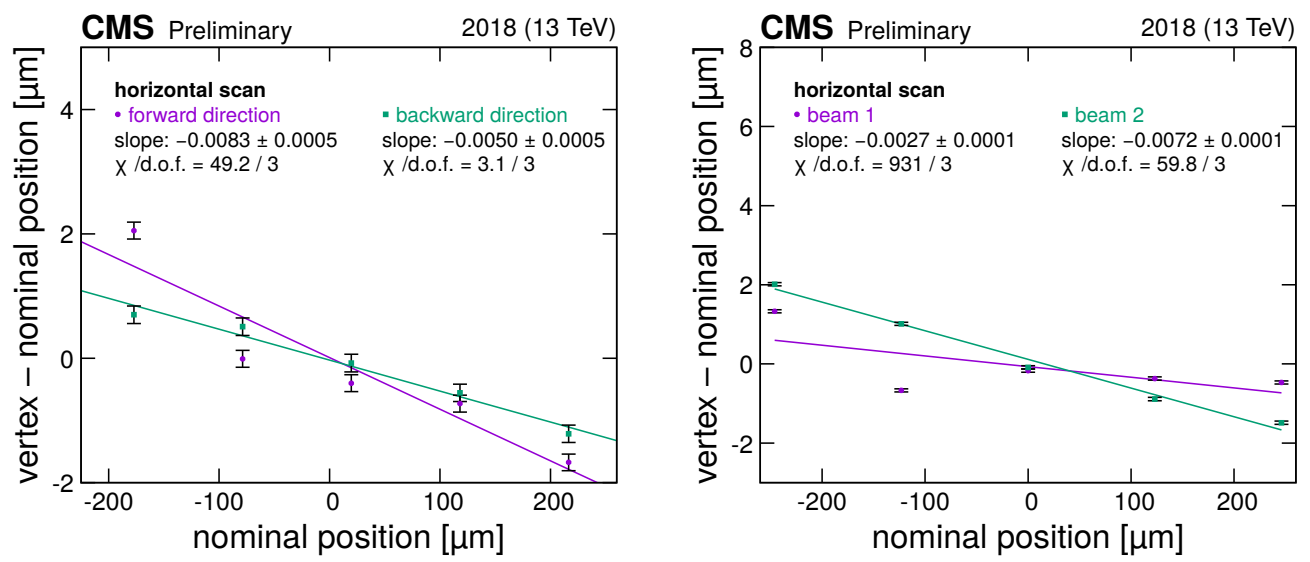

Figure 1: Difference between the reconstructed luminous region position and its nominal position as a function of the nominal position in the constant-separation (left) and variable-seperation (right) LSC scans, in the $x$ plane, when moving the beams in the forward (purple) and backward (green) directions. The plots are fitted with a straight line to derive the correction [2].

The movement of the LHC beam orbits during the vdM scans is measured by two beam position monitoring (BPM) systems, the DOROS and arc BPMs. These "orbit drifts" need to be corrected both in the vdM overlap width measurements and in the LSC procedure.

The assumption that the bunch proton density function is factorizable into independent $x$ and $y$ terms can lead to a biased estimate of the beam overlap integral. This effect is measured using two methods relying on special beam-imaging and offset scans.

The two beams affect each other due to the electromagnetic interaction which is manifested in two ways: the transverse distance between the beams increases which is called "beam-beam deflection", and the shape of the bunches change which is called the "dynamic- $\beta^{*}$ " effect.

Two corrections affect the bunch intensity normalisation, the total beam current measured by the DCCT device and the presence of spurious charges in nominally empty bunch slots (called "ghost charges") and in noncolliding RF buckets of the colliding bunches (called "satellite charges").

After applying the above corrections (and also correcting the luminometer rates for the measured background) and thus calculating $\sigma_{\text {vis }}$, we need to study bunch to bunch and scan to scan in $\sigma_{\text {vis }}$. Also, we consider the agreement among the integrated luminosity measured by the various luminometers for the head-on collision periods in the vdM fill when no scans were taking place to determine the cross-detector consistency of the calibration.

\subsection{Integration uncertainties}

During physics runs, signals coming from out-of-time particles, for example due to the activation of the surrounding detector material, needs to be corrected for. These afterglow corrections are measured using data from empty bunches.

As individual luminometers can be affected by operational issues as well as radiation damage, we need to monitor and if possible correct their stability and linearity. This is done using short vdMlike scans in normal physics conditions, performed at the start and end of fills. After all corrections, 
the luminometer measurements can be compared to each other. Using these comparisons we calculate the cross-detector stability and linearity uncertainties as shown in Fig. 2.
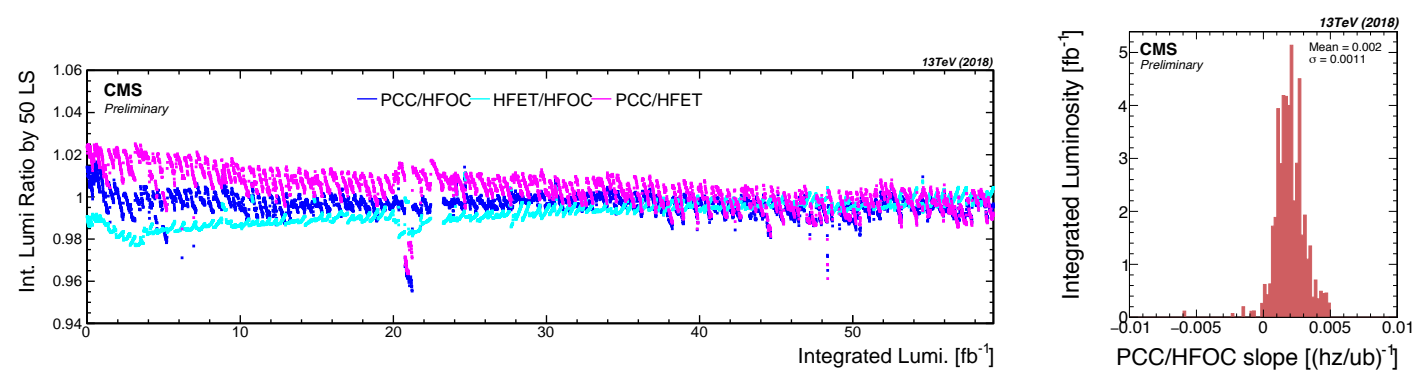

Figure 2: Comparisons of integrated luminosity for different pairs of luminometers (left) and the relative linearity for PCC relative to HFOC (right) in the 2018 pp data set [2].

\section{Summary}

Table 1 lists all systematic uncertainties affecting the 2018 proton-proton luminosity calibration. The total uncertainty is $2.5 \%$ (similar to previous years) and is dominated by the uncertainty due to the $x-y$ factorization assumption and luminometer linearity.

\begin{tabular}{|l|l|c|c|}
\hline & Systematic & Correction [\%] & Uncertainty [\%] \\
\hline \multirow{5}{*}{ Normalization } & Length scale & -0.8 & 0.2 \\
\cline { 2 - 4 } & Orbit drift & 0.2 & 0.1 \\
\cline { 2 - 4 } & $x-y$ nonfactorization & 0.0 & 2.0 \\
\cline { 2 - 4 } & Beam-beam deflection & 1.5 & 0.2 \\
\cline { 2 - 4 } & Dynamic $\beta^{*}$ & -0.5 & 0.2 \\
\cline { 2 - 4 } & Beam current calibration & 2.3 & 0.2 \\
\cline { 2 - 4 } & Ghosts and satellites & 0.4 & 0.1 \\
\cline { 2 - 4 } & Scan to scan variation & - & 0.3 \\
\cline { 2 - 4 } & Bunch to bunch variation & - & 0.1 \\
\cline { 2 - 4 } & Cross-detector consistency & - & 0.5 \\
\cline { 2 - 4 } & Background subtraction & 0.0 to 0.8 & 0.1 \\
\hline \multirow{5}{*}{ Integration } & Afterglow (HFOC) & 0.0 to 4.0 & $0.1 \oplus 0.4$ \\
\cline { 2 - 4 } & Cross-detector stability & - & 0.6 \\
\cline { 2 - 4 } & Linearity & - & 1.1 \\
\cline { 2 - 4 } & CMS deadtime & - & 2.5 \\
\hline & Total & - & 0.1 \\
\hline
\end{tabular}

Table 1: Summary of the systematic uncertainties entering the CMS luminosity measurement for $\sqrt{s}=13$ $\mathrm{TeV}$ pp collisions collected in 2018 [2].

\section{References}

[1] S. Chatrchyan et al. and CMS Collaboration, The CMS experiment at the CERN LHC JINST 3 (2008) S08004 
[2] CMS Collaboration, CMS luminosity measurement for the 2018 data-taking period at $\sqrt{s}=$ $13 \mathrm{TeV}$, CMS-PAS-LUM-18-002 (2019) https: //cds . cern.ch/record/2676164

[3] CMS Collaboration, CMS luminosity measurement for the 2017 data-taking period at $\sqrt{s}=$ $13 \mathrm{TeV}$, CMS-PAS-LUM-17-004 (2018) https://cds . cern.ch/record/2621960

[4] CMS Collaboration, CMS luminosity measurements for the 2016 data-taking period, CMSPAS-LUM-17-001 (2017) https://cds . cern. ch/record/2257069 